\title{
Atividade antibacteriana do óleo essencial de Lavandula hybrida Grosso associado á cefalotina contra cepas de Staphylococcus aureus
}

Antibacterial activity of Lavandula hybrida Grosso essential oil associated with cephalothin against Staphylococcus aureus strains

Actividad antibacteriana del aceite esencial de Lavandula hybrida Grosso asociado con cefalotina contra cepas de Staphylococcus aureus

Maria Tays Pereira Santana ORCID: https://orcid.org/0000-0002-6503-7117 Universidade Federal de Campina Grande, Brasil E-mail: taayssantana@gmail.com

Thallita Alves dos Santos ORCID: https://orcid.org/0000-0002-7100-5023 Universidade Federal de Campina Grande, Brasil E-mail: thallita_28@hotmail.com

Lucas Linhares Gomes ORCID: https://orcid.org/0000-0001-6626-4824 Universidade Federal de Campina Grande, Brasil E-mail: lucaslinharesg@ @otmail.com Maria Angélica Sátyro Gomes Alves ORCID: https://orcid.org/0000-0003-3329-8360 Universidade Federal de Campina Grande, Brasil E-mail: angelicasatyro@hotmail.com

Paula Lima Nogueira ORCID: https://orcid.org/0000-0001-7425-3201 Universidade Federal de Campina Grande, Brasil E-mail: paulalimanogueira@hotmail.com

Maria Vitoria Oliveira Dantas ORCID: https://orcid.org/0000-0001-7663-3793 Universidade Federal de Campina Grande, Brasil E-mail:mvitoria.od@hotmail.com

Quemuel Pereira da Silva ORCID: https://orcid.org/0000-0002-0093-6322 Universidade Federal de Campina Grande, Brasil E-mail: quemuelpereira7@gmail.com

Luanna Abílio Diniz Melquíades de Medeiros ORCID: http://orcid.org/0000-0002-1630-3968 Universidade Federal de Campina Grande, Brasil E-mail: luannaabiliod@gmail.com

Rosana Araújo Rosendo ORCID: https://orcid.org/0000-0002-3795-8832 Universidade Federal de Campina Grande, Brasil E-mail: cesprodonto@hotmail.com

Raline Mendonça dos Anjos ORCID: https://orcid.org/0000-0003-0751-7523

Universidade Federal de Campina Grande, Brasil E-mail: raline.anjos@gmail.com

Marcos Antônio Nóbrega de Sousa ORCID: https://orcid.org/0000-0001-6550-6609 Universidade Federal de Campina Grande (UFCG), Brasil E-mail: marcosandesousa@gmail.com Luciano de Brito Júnior ORCID: https://orcid.org/0000-0002-1787-4316 Universidade Federal de Campina Grande, Brasil E-mail: 1britojunior@hotmail.com

Gymenna Maria Tenório Guênes ORCID: https://orcid.org/0000-0002-5447-0193 Universidade Federal de Campina Grande, Brasil E-mail: gymennat@yahoo.com.br 
Elizandra Silva da Penha

ORCID: https://orcid.org/0000-0001-6264-5232 Universidade Federal de Campina Grande, Brasil E-mail: elizandrapenha@hotmail.com

Manuella Santos Carneiro Almeida ORCID: https://orcid.org/0000-0002-0288-6218 Universidade Federal de Campina Grande, Brasil E-mail: manuellacarneiro@hotmail.com

Aleson Pereira de Sousa

ORCID: https://orcid.org/0000-0002-3430-477X Universidade Federal da Paraíba, Brasil

E-mail: aleson_155@hotmail.com

Abrahão Alves de Oliveira Filho

ORCID: https://orcid.org/0000-0002-7466-9933

Universidade Federal de Campina Grande, Brasil

E-mail: abrahao.farm@gmail.com

\begin{abstract}
Resumo
O presente estudo busca avaliar a atividade antibacteriana do óleo essencial da Lavandula Hybrida Grosso quando associado ao antimicrobiano cefalotina contra cepas de Staphylococcus aureus. O caráter antibacteriano do óleo essencial da Lavandula Hybrida Grosso foi quantificado e qualificado pela técnica de microdiluição em caldo para a obtenção da CIM (Concentração Inibitória Mínima). Em seguida, realizou-se a associação do óleo essencial com o antimicrobiano cefalotina $(10 \mu \mathrm{g} / \mathrm{mL}$ ). A CIM (Concentração Inibitória Minima) foi de $128 \mu \mathrm{g} / \mathrm{mL}$ para a cepa em análise. Quanto a associação, foi observado um efeito sinérgico ao se associar a cefalotina com o óleo essencial da Lavandula Hybrida Grosso. Portanto, observou-se um efeito sinérgico do óleo essencial de Lavandula Hybrida Grosso associado a cefalotina contra cepas patogênicas de S. aureus potencializando sua ação farmacológica antibacteriana. A aplicabilidade da fitoterapia a partir dos compostos provenientes das plantas medicinais se mostra promissora em relação ao tratamento de infecções de caráter bacteriano, colaborando de forma positiva e em conjunto com as terapias convencionais.
\end{abstract}

Palavras-chave: Antimicrobianos; Fitoterapia; Microbiologia.

\begin{abstract}
The present study to evaluate the antibacterial activity of the essential oil of Lavandula Hybrida Grosso when associated with the antimicrobial cephalothin against strains of Staphylococcus aureus. The antibacterial character of the essential oil of Lavandula Hybrida Grosso was quantified and qualified by the broth microdilution technique to obtain the MIC (Minimum Inhibitory Concentration). Then, the essential oil was combined with the cephalothin antimicrobial $(10 \mu \mathrm{g} / \mathrm{mL}$ ). The MIC (Minimum Inhibitory Concentration) was $128 \mu \mathrm{g} / \mathrm{mL}$ for the strain under analysis. As for the association, a synergistic effect was observed when associating cephalothin with the essential oil of Lavandula Hybrida Grosso. Therefore, it was observed a synergistic effect of Lavandula Hybrida Grosso essential oil associated with cephalothin against pathogenic strains of $S$. aureus potentiating its antibacterial pharmacological action. The applicability of phytotherapy based on compounds from medicinal plants is promising in relation to the treatment of bacterial infections, collaborating positively and in conjunction with conventional therapies.
\end{abstract}

Keywords: Antimicrobials; Phytotherapy; Microbiology.

\title{
Resumen
}

El presente estudio para evaluar la actividad antibacteriana del aceite esencial de Lavandula Hybrida Grosso cuando se asocia con el antimicrobiano cefalotina frente a cepas de Staphylococcus aureus. El carácter antibacteriano del aceite esencial de Lavandula Hybrida Grosso fue cuantificado y calificado mediante la técnica de microdilución en caldo para obtener la CIM (Concentración Mínima Inhibitoria). Luego, el aceite esencial se combinó con el antimicrobiano cefalotina $(10 \mu \mathrm{g} / \mathrm{mL}$ ). La MIC (Concentración Inhibitoria Mínima) fue de $128 \mu \mathrm{g} / \mathrm{mL}$ para la cepa bajo análisis. En cuanto a la asociación, se observó un efecto sinérgico al asociar cefalotina con el aceite esencial de Lavandula Hybrida Grosso. Por tanto, se observó un efecto sinérgico del aceite esencial de Lavandula Hybrida Grosso asociado a cefalotina frente a cepas patógenas de $S$. aureus potenciando su acción farmacológica antibacteriana. La aplicabilidad de la fitoterapia basada en compuestos de plantas medicinales es prometedora en relación al tratamiento de infecciones bacterianas, colaborando positivamente y en conjunto con terapias convencionales.

Palabras clave: Antimicrobianos; Fitoterapia; Microbiologia. 


\section{Introdução}

A fitoterapia é um recurso terapêutico que se caracteriza pelo uso de plantas medicinais em suas diversas preparações, constituindo uma modalidade de terapia integrativa e complementar diante dos problemas de saúde enfrentados pela sociedade, seu uso tem tido um crescimento significativo na população de diversos países (Bruning et al., 2012; Santos et al., 2011). Um dos motivos que influenciaram neste crescente interesse pela fitoterapia foi ascensão na área científica, que tornou possível a manipulação de fitoterápicos comprovadamente seguros e eficazes (Bruning et al., 2012).

Os fitoterápicos constituem atualmente uma classe de medicamento com grande potencial de desenvolvimento no Brasil, o crescente uso de plantas medicinais pela população brasileira e por profissionais da saúde estimulam áreas como a farmacognosia na investigação e no desenvolvimento de produtos com intuito de implementar medidas curativas e preventivas (Viana, 2011; Ferreira et al., 2014).

É milenar o uso de medicamentos à base de produtos naturais para o tratamento ou para a prevenção de enfermidades principalmente aquelas de origem bacteriana, a qual a elevada resistência dos microrganismos patogênicos aos antimicrobianos, associado ao seu uso de maneira irracional, gerando uma preocupação cada vez maior, dessa forma, é necessária que além da criação de novos antimicrobianos sintéticos, o uso da fitoterapia como via alternativa de tratamento possa de alguma forma minimizar esse problema (Dietz et al., 2016; Van Dooren et al., 2017; Hameed et al., 2017).

Dessa forma são relevantes os estudos que mostram a diversidade de plantas medicinais com atividade antibacteriana, tais como a Romã (Punica granatum) tem o seu uso muito difundido na odontologia, e trabalhos realizados demonstraram que essa espécie vegetal tem ação bactericida e bacteriostática sobre bactérias Gram-positivas e Gram-negativas, constituintes do biofilme dental, podendo ser utilizada para casos de periodontites, como antioxidantes e de estomatites, como antisséptico (Soares et al., 2007).

Outros produtos amplamente utilizados na cultura popular são o alho (Allium sativum), que demonstrou atividade contra bactérias orais, sendo que os resultados de alguns estudos mostraram que há uma diminuição do número de bactérias quando os pacientes são submetidos ao uso de enxaguatórios contendo este esse produto e a aroeira (Schinus terenithiofolius) possui ação apresenta atividade bactericida e bacteriostática sobre Streptococus mutans, Streptococus mitis, Streptococus sobrinus, Streptococus sanguis, (Lactobacillus casei) (Salgado et al., 2006).

Estudos recentes realizados por Medeiros et al. (2019) avaliou o potencial do óleo essencial de Lavandula Hybrida Grosso frente a cepas patogênicas de Escherichia coli, Bajalan et al. (2017) demostrou que o óleo essencial da Lavanda possui atividade antibacteriana contra Streptococcus agalactiae, Klebsiella pneumoniae e Staphylococcus aureus. De acordo com o exposto anteriormente, este estudo tem como objetivo avaliar a atividade antibacteriana do óleo essencial da Lavandula Hybrida Grosso quando associado ao antimicrobiano cefalotina contra cepas de Staphylococcus aureus (S. aureus).

\section{Metodologia}

\subsection{Substâncias-teste}

O óleo essencial de Lavandula Hybrida Grosso foi adquirido da Indústria Via Aroma® (Porto Alegre - RS). Para a realização dos ensaios farmacológicos, a substância foi solubilizada em DMSO e diluída em água destilada. A concentração de DMSO (dimetilsulfóxido) utilizada foi inferior a $0,1 \% \mathrm{v} / \mathrm{v}$.

\subsection{Antimicrobiano sintético}

Foi selecionado no presente estudo o antibiótico cefalotina $(10 \mu \mathrm{g} / \mathrm{mL})$. A concentração contida no disco de antibiótico é uma concentração padrão determinada pelo CLSI (2003). O estudo de observação de sensibilidade da cepa 
bacteriana frente antibiótico foi realizado através da técnica de difusão em meio sólido utilizando-se discos de papel de filtro (Newprov®) (Vandepitte et al., 1994).

\subsection{Espécie Bacteriana e Meio de cultura}

O microorganismo utilizado foi a cepa clínica de S. aureus (SA 101), proveniente do Laboratório de Microbiologia da Unidade Acadêmica de Ciências Biológicas do Centro de Saúde e Tecnologia Rural da Universidade Federal de Campina Grande.

A cepa foi mantida em meio Ágar Muller Hinton (AMH) a uma temperatura de $4{ }^{\circ} \mathrm{C}$, sendo utilizado para os ensaios repiques de 24 horas em AMH incubados a $35^{\circ} \mathrm{C}$. No estudo da atividade antimicrobiana foi utilizado um inóculo bacteriano de aproximadamente $1,5 \times 10^{8} \mathrm{UFC} / \mathrm{mL}$ padronizado de acordo com a turbidez do tubo 0,5 da escala de McFarland (Cleeland \& Squires, 1991).

\subsection{Determinação da Concentração Inibitória Mínima (CIM)}

A concentração inibitória mínima do óleo foi determinada pela técnica de microdiluição em caldo (Cleeland \& Squires, 1991). Foi utilizada uma placa de 96 orifícios estéreis e com tampa. Em cada orifício da placa, foi adicionado $100 \mu \mathrm{L}$ do meio líquido Muller Hinton (MH) duplamente concentrado. Em seguida, $100 \mu \mathrm{L}$ da emulsão do óleo na concentração inicial de $2048 \mu \mathrm{g} / \mathrm{mL}$ (também duplamente concentrado), foram dispensados nas cavidades da primeira linha da placa. E por meio de uma diluição seriada em razão de dois, foram obtidas as concentrações de 1024, 512, 256, 128, 64, 32, 16, 8 e $4 \mu \mathrm{g} / \mathrm{mL}$, de modo que na primeira linha da placa encontra-se a maior concentração e na última, a menor concentração. Por fim, foi adicionado $10 \mu \mathrm{L}$ do inóculo de aproximadamente 1-5 x 10 $\mathrm{UFC} / \mathrm{mL}$ da espécie bacteriana nas cavidades.

Paralelamente, foi realizado o mesmo ensaio com o antibacteriano cloranfenicol, o controle positivo. Um controle de microrganismo foi realizado colocando-se nas cavidades $100 \mu \mathrm{L}$ do mesmo $\mathrm{MH}$ duplamente concentrado, $100 \mu \mathrm{L}$ de água destilada estéril e $10 \mu \mathrm{L}$ do inóculo de cada espécie. Para verificar a ausência de interferência no resultado pelos solventes utilizados na preparação da emulsão, no caso o DMSO, foi feito um controle no qual foi colocado nas cavidades $100 \mu \mathrm{L}$ do caldo duplamente concentrado, $100 \mu \mathrm{L}$ de DMSO e $10 \mu \mathrm{L}$ da suspensão bacteriana. Um controle de esterilidade do meio também foi realizado, onde foi colocado $200 \mu \mathrm{L}$ do $\mathrm{MH}$ em um orifício sem a suspensão das bactérias.

A placa foi assepticamente fechada e incubada a $35^{\circ} \mathrm{C}$ por $24 \mathrm{hs}$ em seguida foi realizada a leitura da CIM para o monoterpeno e o antibacteriano a qual foi definida como a menor concentração capaz de inibir visualmente o crescimento bacteriano verificado nos orifícios quando comparado com o crescimento controle.

\subsection{Interferência do óleo essencial sobre o efeito de antimicrobianos sintético}

O estudo da interferência do óleo essencial sobre a efetividade dos antimicrobianos foi realizado através da técnica de difusão em meio sólido utilizando disco de cefalotina. O disco contendo o antimicrobiano na sua respectiva concentração foi embebido com $20 \mu \mathrm{L}$ da CIM do óleo essencial, e em seguida colocado em placa de Petri estéril contendo ágar Muller-Hinton inoculado com $1 \mathrm{~mL}$ da suspensão bacteriana. Após incubação das placas a $37^{\circ} \mathrm{C}$ por 48 horas, foi observada a interferência da CIM óleo essencial sobre o efeito do antimicrobiano sobre a cepa bacteriana ensaiada. Foi considerado como efeito sinérgico, quando o halo de inibição do crescimento microbiano formado pela associação teve um diâmetro $\geq$ que $2 \mathrm{~mm}$, quando comparado com o halo de inibição formado pela ação do antimicrobiano isoladamente. Quando a formação de halo de inibição decorrente da associação fosse de um diâmetro menor daquele desenvolvido pela ação isolada do antimicrobiano, era considerado efeito antagônico. Foi considerado como efeito indiferente, quando o de halo de inibição consequente à associação 
tivesse um diâmetro igual àquele consequente da aplicação isolada do antimicrobiano (Cleeland \& Squires, 1991). Todos os ensaios foram realizados em duplicata.

\section{Resultados e Discussão}

A análise dos resultados mostrou que a CIM (Concentração Inibitória Mínima) foi de $128 \mu \mathrm{g} / \mathrm{mL}$ para a cepa analisada. Em relação á associação do óleo essencial de Lavandula Hybrida Grosso com o antibiótico cefalotina, mostrou um aumento do tamanho do halo de inibição do antimicrobiano, sendo esse resultado obtido a partir da análise comparativa do halo de inibição na presença do antibiótico com halo de inibição na presença do antibiótico associado com o óleo essencial, como pode ser visto na Tabela 1.

Tabela 1. Interferência do óleo essencial de Lavandula Hybrida Grosso com o antibiótico sintético.

\begin{tabular}{c|c}
\hline Antibiótico & Microrganismo \\
\hline Cefalotina & S. aureus \\
& SA 101 \\
HIATB & $3,0 \mathrm{~mm}$ \\
HIATB + OE & $3,6 \mathrm{~mm} \square$ \\
\hline
\end{tabular}

Legenda: HIATB: halo de inibição na presença do antibiótico. OE: óleo essencial. Efeito sinérgico ( $\square$ ); efeito antagônico ( $\downarrow$ ); efeito indiferente $(*)$. Fonte: Autores.

Dessa forma, observou-se que o óleo essencial de Lavandula Hybrida Grosso quando associado ao antimicrobiano cefalotina apresentou uma excelente ação antibacteriana, efeito sinérgico, ao observar um aumento maior que $2 \mathrm{~mm}$ do halo de inibição. De acordo com os critérios estabelecidos por Cleeland e Squires (1991).

Atualmente, o uso de plantas medicinais constitui uma prática generalizada na medicina caseira, podendo ser considerada como o resultado do acúmulo de conhecimento da ação de vegetais por diversos grupos éticos (Reginatto, 2011).

Dentre os derivados presentes nas plantas tem-se, os óleos essenciais que consistem em misturas complexas de compostos naturais voláteis, em geral odoríferos, presentes em determinadas espécies vegetais, sendo provenientes do seu metabolismo secundário, a análise química desses produtos tem revelado a presença majoritária de monoterpenos, sesquiterpenos e arilpropanóides (Moreira et al., 2001).

Os óleos essenciais são conhecidos pela fragrância e propriedades fungicidas, antivirais, bactericidas e medicinais e podem ser utilizados como antimicrobianos, analgésicos, sedativos e anti-inflamatório (Bakkali et al., 2008).

Dentre essas propriedades a capacidade bactericida desses compostos tem colaborado de forma positiva no tratamento de doenças bacteriana, podendo citar os óleos essenciais presentes nas plantas da família Lamiaceae, a exemplo tem-se a Lavandula Hybrida Grosso que evidenciou a presença de atividade antibacteriana contra E. coli por Medeiros et al. (2019), efeito sobre as cepas S. agalactiae, K. pneumoniae e S. aureus por Bajalan et al. (2017), a investigação sobre a interação de produtos naturais com fármacos utilizados na terapêutica contribui de forma significativa para clínica.

Os produtos naturais que apresentam potencial terapêutico utilizados no cuidado da saúde tradicional constituem uma importante fonte de novos compostos biologicamente ativos, o que fortalece a capacidade de desenvolver novos medicamentos a partir de plantas medicinais e seus derivados, que tragam a mesma eficácia, segurança e biocompatibilidade (Samuelsson \& Bohlin, 2017). 
Dessa forma, é notória a motivação de estudos voltados a análise de plantas e seus derivados, além da observação de associações entre esses derivados e antimicrobianos sintéticos, visando seu emprego como fonte de recursos terapêuticos e como um imenso provedor de biomoléculas (Foglio et al., 2006).

Portanto, é frequente a realização de estudos voltados a análise de associações de substâncias naturais com medicamentos convencionais, como por exemplo o estudo de Oliveira et al. (2006) o qual analisou quatro óleos essenciais de diferentes espécies vegetais associado com cefalotina contra S. aureus, nessa análise observou-se que os óleos Lippia sidoides, Plectranthus amboinicus e Conyza bonariensis apresentaram efeito sinérgico, enquanto que óleo Eucalyptus citriodora demonstrou efeito indiferente, demostrando assim que a associação de produtos naturais com antibióticos sintéticos consiste em uma importante opção terapêutica no combate a resistência de bactérias do gênero Staphylococcus.

\section{Conclusão}

O estudo conclui que a interação do óleo essencial de Lavandula Hybrida Grosso associado a cefalotina contra cepas patogênicas de $S$. aureus possui ação de efeito sinérgico potencializando o desempenho da cefalotina contra as bactérias. A aplicabilidade da fitoterapia a partir dos compostos provenientes das plantas medicinais se mostra promissora em relação ao tratamento de infecções de caráter bacteriano, colaborando de forma positiva e em conjunto com as terapias convencionais. Entretanto é importante o incentivo dos estudos da área de pesquisa voltados a uma análise cada vez mais detalhada sobre esses compostos naturais e sua interação com o organismo para que sua comercialização seja segura e eficaz.

\section{Referências}

Bajalan, I., Rouzbahani, R., Pirbalouti, A. G., \& Maggi, F. (2017). Chemical composition and antibacterial activity of Iranian Lavandula $\times$ hybrida. Chemistry \& biodiversity, 14(7), e1700064.

Bakkali, F., Averbeck, S., Averbeck, D., \& Idaomar, M. (2008). Biological effects of essential oils-a review. Food and chemical toxicology, 46(2), 446-475.

Bruning, M. C. R., Mosegui, G. B. G., \& Vianna, C. M. D. M. (2012). A utilização da fitoterapia e de plantas medicinais em unidades básicas de saúde nos municípios de Cascavel e Foz do Iguaçu-Paraná: a visão dos profissionais de saúde. Ciência \& saúde coletiva, 17, $2675-2685$.

Cleeland, R., \& Squires, E. (1991). Antibiotics in laboratory medicine. Willians. \& Wikins.

Clinical and Laboratory Standard Institute (CLSI) - Approved standard M2-A7: Performance standards for antimicrobial disk susceptibility tests. Seventh edition. Wayne-PA, 2003.

Dietz, B. M., Hajirahimkhan, A., Dunlap, T. L., \& Bolton, J. L. (2016). Botanicals and their bioactive phytochemicals for women's health. Pharmacological reviews, 68(4), 1026-1073.

Ferreira, T. S., Moreira, C. Z., Cária, N. Z., Victoriano, G., Silva Jr, W. F., \& Magalhães, J. C. (2014). Fitoterapia: introdução a sua história, uso e aplicação. Revista Brasileira de Plantas Medicinais, 16(2), 290-298.

Foglio, M. A., Queiroga, C. L., Sousa, I. D. O., \& Rodrigues, R. A. F. (2006). Plantas medicinais como fonte de recursos terapêuticos: um modelo multidisciplinar. Construindo a história dos produtos naturais, 7, 1-8.

Hameed, I. H., Al-Rubaye, A. F., \& Kadhim, M. J. (2017). Antimicrobial Activity of Medicinal Plants and Urinary Tract Infections. International Journal of Pharmaceutical and Clinical Research, 9(1), 44-50.

Medeiros, M. A. A., Souza Alves, M., Simão, K. D. L. A., Pereira, C. T., Simão, B. D. L. A., Pessoa, H. L. F., Diniz, M. F. F. M., Oliveira Filho, A. A. (2019). Avaliação da atividade antibacteriana do óleo essencial de Lavandula hybrida Grosso contra cepas de Escherichia coli. Revista Saúde \& Ciência Online, 8(2), 58-65.

Moreira, D. L., Souza, P. O., Kaplan, M. A. C., Pereira, N. A., Cardoso, G. L., \& Guimarães, E. F. (2001). Effect of leaf essential oil from Piper solmsianum C. DC. in mice behaviour. Anais da Academia Brasileira de Ciências, 73(1), 33-57.

Oliveira, R. A., Lima, E. O., Vieira, W. L., Freire, K. R. L., Trajano, V. N., Lima, I. O., \& Silva-Filho, R. N. (2006). Estudo da interferência de óleos essenciais sobre a atividade de alguns antibióticos usados na clínica. Revista Brasileira de Farmacognosia, 16(1), 77-82.

Salgado, A. D. Y., Maia, J. L., Pereira, S. L. D. S., Lemos, T. L. G. D., \& Mota, O. M. D. L. (2006). Antiplaque and antigingivitis effects of a gel containing Punica granatum Linn extract: a double-blind clinical study in humans. Journal of Applied Oral Science, 14(3), $162-166$.

Samuelsson, G., \& Bohlin, L. (2017). Drugs of natural origin: a treatise of pharmacognosy (7a ed.). CRC Press Inc. 
Research, Society and Development, v. 10, n. 2, e47110212682, 2021

(CC BY 4.0) | ISSN 2525-3409 | DOI: http://dx.doi.org/10.33448/rsd-v10i2.12682

Santos, R. L., Guimaraes, G. P., Nobre, M. S. D. C., \& Portela, A. D. S. (2011). Análise sobre a fitoterapia como prática integrativa no Sistema Único de Saúde. Revista Brasileira de Plantas Medicinais, 13(4), 486-491.

Sousa Soares, D. G., de OLIVEIRA, C. B., Leal, C., Drumond, M. R. R. S., \& Padilha, W. W. N. (2007). Atividade Antibacteriana in vitro da Tintura de Aroeira (Schinus terebinthifolius) na Descontaminação de Escovas Dentais Contaminadas pelo S. mutans. Pesquisa Brasileira em Odontopediatria e Clínica Integrada, 7(3), 253-527.

van Dooren, I., Foubert, K., Theunis, M., Naessens, T., Pieters, L., \& Apers, S. (2018). Advantages of a validated UPLC-MS/MS standard addition method for the quantification of A-type dimeric and trimeric proanthocyanidins in cranberry extracts in comparison with well-known quantification methods. Journal of pharmaceutical and biomedical analysis, 148, 32-41.

Vandepitte, J., Engbaek, K., Piot, P., Heuck, C. C., \& Levanon, Y. (1994). Procedimentos laboratoriais em bacteriologia clínica. In Procedimentos laboratoriais em bacteriologia clínica (pp. 122-122).

Viana, M. (2011). Medicamentos fitoterápicos. Rev. Rio Pharma, 41-41. 